\title{
Alcohol drinking, tobacco smoking and subtypes of haematological malignancy in the UK Million Women Study
}

\author{
ME Kroll', , F Murphy', K Pirie', GK Reeves', J Green'and V Beral' for the Million Women Study Collaborators \\ 'Cancer Epidemiology Unit, Nuffield Department of Medicine, University of Oxford, Richard Doll Building, Roosevelt Drive, Oxford OX3 7LF, UK
}

\begin{abstract}
BACKGROUND: Previous research suggests associations of lower alcohol intake and higher tobacco consumption with increased risks of haematological malignancy. The prospective Million Women Study provides sufficient power for reliable estimates of subtype-specific associations in women.

METHODS: Approximately 1.3 million middle-aged women were recruited in the United Kingdom during 1996-200I and followed for death, emigration and cancer registration until 2009 (mean 10.3 years per woman); potential risk factors were assessed by questionnaire. Adjusted relative risks were estimated by Cox regression.

RESULTS: During follow-up, 9162 incident cases of haematological malignancy were recorded, including 7047 lymphoid and 2072 myeloid cancers. Among predominantly moderate alcohol drinkers, higher intake was associated with lower risk of lymphoid malignancies, in particular diffuse large B-cell lymphoma (relative risk 0.85 per $10 \mathrm{~g}$ alcohol per day (95\% confidence interval 0.75-0.96)), follicular lymphoma (0.86 (0.76-0.98)) and plasma cell neoplasms (0.86 (0.77-0.96)). Among never- and current smokers, higher cigarette consumption was associated with increased risk of Hodgkin lymphoma ( 1.45 per 10 cigarettes per day (1.22-1.72)), mature T-cell malignancies (1.38 (1.10-1.73)) and myeloproliferative/myelodysplastic disease (1.42 (1.31-1.55)).

CONCLUSION: These findings confirm and extend existing evidence for associations of subtypes of haematological malignancy with two common exposures in women.
\end{abstract}

British Journal of Cancer (2012) 107, 879-887. doi:I0.1038/bjc.2012.333 www.bjcancer.com

Published online 9 August 2012

(c) 2012 Cancer Research UK

Keywords: alcohol; smoking; lymphoma; leukaemia; plasma cell neoplasms; myelodysplastic/myeloproliferative neoplasms

Haematological malignancies are cancers that originate from lymphoid or myeloid cells and affect blood, bone marrow and lymph nodes. The tenth revision of the International Classification of Diseases (ICD-10) groups cases primarily by clinical presentation (leukaemia, myeloma or lymphoma). In contrast, the third edition of the International Classification of Diseases for Oncology (ICD-O-3) groups haematological malignancies primarily by cell lineage (lymphoid or myeloid), and includes some myeloid neoplasms that are not coded as malignant in ICD-10 (Jaffe et al, 2001; Swerdlow, 2008). A hierarchical classification based on ICD$\mathrm{O}-3$ has been used in recent international collaborative studies (Morton et al, 2007; Sant et al, 2010; Turner et al, 2010).

Alcohol drinking and tobacco smoking are modifiable exposures that are widespread in developed countries. Both are known to be associated with risks of certain types of haematological malignancy. Several recent cohort studies have reported decreasing trends in risk of non-Hodgkin lymphoma (NHL) and/or diffuse large B-cell lymphoma (a major subtype of NHL) with increasing alcohol intake among drinkers (Lim et al, 2007; Allen et al, 2009; Kanda et al, 2010; Troy et al, 2010). Smoking is considered to be causally related to myeloid leukaemia in adults (IARC, 2002), and (comparing current with never-smokers) has been associated with increased risk of Hodgkin lymphoma, acute myeloid leukaemia and myelodysplastic syndromes in recent cohort (Fernberg et al,

*Correspondence: Dr ME Kroll; E-mail: mary.kroll@ceu.ox.ac.uk Received 4 April 2012; revised 21 June 2012; accepted 24 June 2012; published online 9 August 2012
2007; Lim et al, 2007; Nieters et al, 2008; Ma et al, 2009, 2010) and case-control (Kasim et al, 2005; Besson et al, 2006a) studies. However, haematological malignancies are probably heterogeneous in aetiology, and much of the evidence for subtype-specific associations remains inconclusive, perhaps reflecting inconsistent exposure classifications, or relatively small study sizes.

We examined association of subtypes of haematological malignancy with alcohol drinking and tobacco smoking in the prospective Million Women Study. To aid comparison with previous research, we report findings based on both ICD-O-3 and ICD-10. The large size of this cohort provides sufficient power to estimate risk for relatively rare subtypes.

\section{MATERIALS AND METHODS}

\section{Definitions}

The Million Women Study has been described elsewhere (Reeves et al, 2007). Between 1996 and 2001, with appropriate ethical approval, 1.3 million middle-aged women were recruited through breast cancer screening clinics in the United Kingdom. Participants gave written informed consent, and completed questionnaires recording personal and lifestyle characteristics (available at www.millionwomenstudy.org). By linkage to the National Health Service Central Registers, participants are followed for death, emigration and cancer registration. Each incident neoplasm is coded using the combination of a disease code from ICD-10 and a morphology 
code from either the second or the third (ICD-O-3) edition of the International Classification of Diseases for Oncology.

For this analysis, haematological neoplasms were defined by the following ICD-10 codes: C81-C96 (malignant neoplasms of lymphoid, haematopoietic and related tissue), D45 (polycythemia vera), D46 (myelodysplastic syndromes) and D47 (other neoplasms of uncertain or unknown behaviour of lymphoid, haematopoietic and related tissue). Women were excluded if they had been diagnosed before recruitment with any haematological neoplasm (as defined above), or cancer of any other site except non-melanoma skin cancer (all other ICD-10 C codes except C44), or in situ breast carcinoma (ICD-10 D05), using equivalent definitions from earlier standard coding systems where necessary. For the remaining women, observation extended from the date of recruitment to the date of death, emigration, diagnosis with any of the neoplasms listed in the exclusion criteria, or end of follow-up, whichever occurred first. Follow-up ended on 31 December 2008 for Scotland and the North West (Merseyside and Cheshire) cancer registry region, and 31 December 2009 elsewhere.

Morphology codes for incident haematological neoplasms were converted from the second to the third edition as necessary (National Cancer Institute, 2011). Where the morphology code was uninformative $(80001 / 80003, N=115)$ or discrepant $(N=3)$, it was changed to match the ICD-10 code, unless both were non-specific $(N=10)$. Malignant disease was defined and classified in two different ways (Table 1). ICD-O-3 morphology codes with fifth digit 3 (malignant) and first three digits in the range 959-998 (haematological neoplasms), except 975 (histiocytic and dendritic neoplasms, $N=2$ ), were grouped using a classification adapted from the InterLymph hierarchical scheme for epidemiological research (Turner et al, 2010) and the Haemacare project (Sant et al, 2010). Cases were grouped primarily by cell lineage. Subtypes of mature B-cell lymphoid malignancy included plasma cell neoplasms and 'CLL/SLL' (cases coded as either chronic lymphocytic leukaemia or small lymphocytic lymphoma, now considered to be a single disease). Hodgkin lymphoma formed a separate subtype of lymphoid malignancy. Myeloid malignancies were divided into two subtypes: acute myeloid leukaemia and 'myeloproliferative/myelodysplastic disease' (myeloproliferative and myelodysplastic neoplasms, including chronic myeloid leukaemia). For comparison, cases with ICD-10 codes C81-C96 were classified as Hodgkin lymphoma (C81), NHL (C82-C85, C96), 'myeloma' (multiple myeloma, plasma cell neoplasms and malignant immunoproliferative diseases (C88, C90)) and leukaemia (C91-C95).

\section{Statistical analysis}

Relative risk was estimated by Cox regression, taking attained age as the underlying time variable, and stratifying by cancer registry region of residence at recruitment (i.e. assuming equal coefficients across strata but with a baseline hazard unique to each stratum) (StataCorp., 2009). The proportional hazards assumption was examined using Schoenfeld residuals and found acceptable. To assess the possibility that associations might reflect lifestyle changes caused by subclinical disease (reverse causation), all analyses were repeated without the first 3 years of follow-up. All statistical tests were two-sided and used the 5\% significance level.

Categorical exposure measures were derived from information given on the questionnaire completed by each woman at the time of recruitment to the study, as follows: current weekly alcohol consumption (none, $0.5-<3,3-<7, \geqslant 7$ drinks, in units equivalent to approximately $10 \mathrm{~g}$ of pure alcohol); tobacco smoking (past, never, current $<15$ cigarettes per day, current $\geqslant 15$ cigarettes per day); socioeconomic status (within-study quintiles of the 1991 Townsend deprivation index for the census enumeration district or output area containing the woman's home address at recruitment

Table I Number of women diagnosed with haematological neoplasms during follow-up: cross-classification by ICD-O-3 and ICD- I0

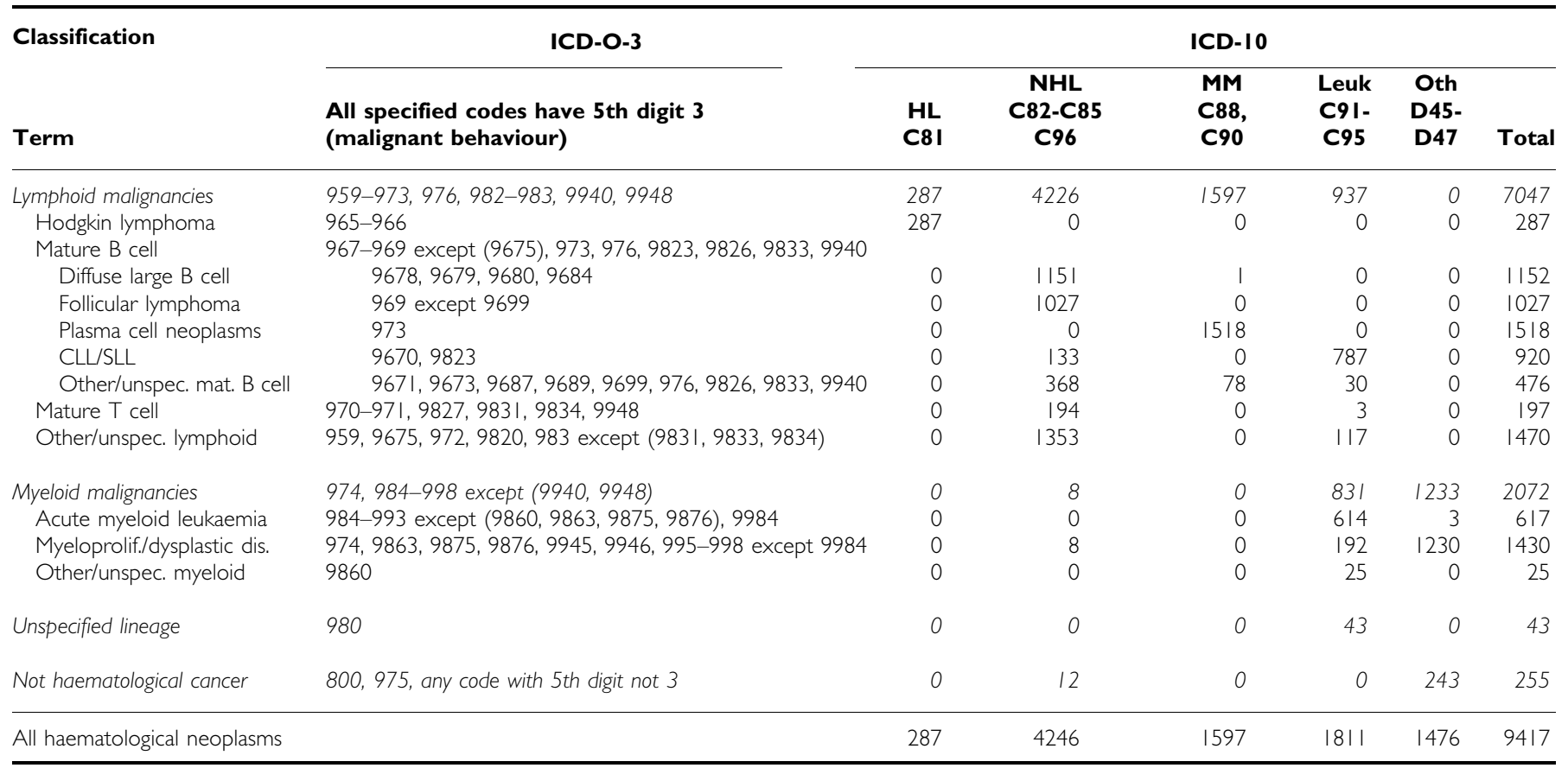

Abbreviations: CLL/SLL = chronic lymphocytic leukaemia/small lymphocytic lymphoma; HL=Hodgkin lymphoma; ICD-O-3=International Classification of Diseases for Oncology 3rd edition; ICD-10=International Classification of Diseases IOth revision; Leuk=leukaemia; $M M=$ myeloma (multiple myeloma, plasma cell neoplasms and malignant immunoproliferative diseases); Myeloprolif/dysplastic dis. = myeloproliferative/myelodysplastic disease (including chronic myeloid leukaemia); NHL = non-Hodgkin lymphoma; Oth = other haematological neoplasms. 
(Townsend, 1988)); body mass index $\left(<25,25-<30, \geqslant 30 \mathrm{~kg} \mathrm{~m}^{-2}\right)$; height $(<160,160-<165, \geqslant 165 \mathrm{~cm})$. The questions relevant to smoking habits were 'About how many cigarettes do you smoke on average each day, now?' and 'Are you an ex-smoker?' (yes/no). Body mass index (Reeves et al, 2007) and height (Green et al, 2011) were known to be associated with risks of haematological malignancy in this cohort.

In turn, each of the two factors of interest (alcohol and smoking) was treated as the main explanatory variable, with all the other exposure measures acting as adjustment factors. Women with missing information for the explanatory variable were excluded from that analysis; those with missing information for an adjustment factor were included as a separate category of the adjustment factor. Trends were assessed by allocating a score to each category of the explanatory variable, and fitting log-linear models to the change in hazard ratio per unit increase in score. Current nondrinkers were excluded from the alcohol trend model, and exsmokers from the smoking trend model, because reasons for abstention might include ill-health. As an approximate correction for regression dilution bias (Macmahon et al, 1990), each category of drinking and smoking was scored as the mean daily intake reported at re-survey approximately 3 years after recruitment among women in that category: drinks in units of approximately $10 \mathrm{~g}$ pure alcohol per day $(0.26,0.75,1.63)$ and smoking in multiples of 10 cigarettes per day $(0,1.10,2.00)$, scoring selfreported never-smokers at recruitment as zero. Heterogeneity of trends between diagnostic groups was assessed by a $\chi^{2}$ contrast test (Smith-Warner et al, 2006).

\section{RESULTS}

\section{Descriptive statistics}

The number of women who were eligible for these analyses was 1319121 , after excluding 45035 with neoplasms diagnosed before recruitment. On average, women were aged 56.6 years at recruitment, and contributed 10.3 person-years to the analyses.

The number of women diagnosed with haematological malignancies during follow-up was 9162 according to the hierarchical classification based on ICD-O-3, and 7941 according to the simple ICD-10 grouping (Table 1); the main reason for the difference was that clinical behaviour for 1230 cases of myeloproliferative/ myelodysplastic disease was treated as malignant in ICD-O-3 but uncertain or unknown in ICD-10. Of the 5093 mature B-cell cases,
$2679(53 \%)$ were coded in ICD-10 as NHL, 1597 (31\%) as myeloma and $817(16 \%)$ as leukaemia.

Information on alcohol consumption at recruitment was obtained from 1308786 women (99\%), of whom 994030 (76\%) reported $\geqslant 0.5$ drinks per week (Table 2). Among drinkers at recruitment, the mean intake reported at re-survey was 5.6 drinks per week, a moderate level by national standards (Office for National Statistics, 2003). Of the 1241605 women (94\%) who could be classified as never, current or past smokers at recruitment, $21 \%$ (255 148) were current smokers and 28\% (352493) were past smokers. The measure of socioeconomic status was available for 1309534 women (99\%). Height was reported by 98\% of women, and both height and weight (enabling calculation of body mass index) by $95 \%$.

Socioeconomic status, body mass index, height and age varied with alcohol and tobacco intake (Table 2). The proportion of participants with relatively low socioeconomic status was smaller for drinkers than non-drinkers, and greater for current smokers than never-smokers. On average, drinkers were slightly leaner, taller and younger than non-drinkers, and current smokers were slightly leaner, shorter and younger than never-smokers.

\section{Alcohol}

Using the ICD-O-3 classification, and taking occasional drinkers $(0.5-<3$ drinks per week) as the reference group, the estimated relative risk of haematological malignancy was 0.90 (95\% confidence interval $0.85-0.95$ ) for $\geqslant 7$ drinks per week (Table 3). Among drinkers, there was a statistically significant decreasing trend with increasing alcohol intake $\left(P_{\text {trend }}<0.001\right.$; Table 3$)$; the estimated relative risk for an increase of $10 \mathrm{~g}$ per day was $0.92(0.89-0.96)$ (Figure 1). In more detail, there was a statistically significant decreasing trend for the lymphoid subgroup $\left(P_{\text {trend }}<0.001\right)$ and no apparent trend for the myeloid subgroup, although the test for heterogeneity between lymphoid and myeloid trends was not statistically significant $\left(P_{\text {het }}=0.09\right.$; Figure 1$)$. Among specified subtypes of lymphoid malignancy, there was a statistically significant trend only for mature B-cell disease $\left(P_{\text {trend }}<0.001\right)$, within which there were similar significant decreasing trends for diffuse large B-cell lymphoma, follicular lymphoma and plasma cell neoplasms, but not CLL/SLL; the test for heterogeneity was not statistically significant $\left(P_{\text {het }}=0.1\right)$. For Hodgkin lymphoma, the risk was significantly higher in non-drinkers than in occasional drinkers (relative risk 1.70

Table 2 Characteristics of the women included in these analyses

\begin{tabular}{|c|c|c|c|c|c|c|}
\hline & \multicolumn{2}{|c|}{ Alcohol } & \multicolumn{3}{|c|}{ Smoking } & \multirow[b]{2}{*}{ All women } \\
\hline & Non-drinkers & Drinkers $^{a}$ & Past & Never & Current & \\
\hline Number of women & 314756 & 994030 & 352493 & 633964 & $255 \mid 48$ & 1319121 \\
\hline \multicolumn{7}{|l|}{ Characteristics at recruitment } \\
\hline$\%$ Drinkers $^{\mathrm{a}}$ & - & - & 81 & 76 & 70 & 76 \\
\hline$\%$ Current smokers & 26 & 19 & - & - & - & 21 \\
\hline$\%$ Lower socioeconomic status $^{b}$ & 45 & 30 & 33 & 27 & 48 & 33 \\
\hline Body mass index $\left(\mathrm{kg} \mathrm{m}^{-2}\right)$ : mean (s.d.) & $27.2(5.4)$ & $25.9(4.4)$ & $26.7(4.8)$ & $26.2(4.6)$ & $25.6(4.5)$ & $26.2(4.7)$ \\
\hline Height (cm): mean (s.d.) & $161.2(6.9)$ & $162.2(6.7)$ & $162.3(6.7)$ & $162.0(6.7)$ & $161.5(6.8)$ & $162.0(6.7)$ \\
\hline Age (years): mean (s.d.) & $57.3(4.9)$ & $56.4(4.8)$ & $56.8(4.9)$ & $56.8(4.9)$ & $55.8(4.5)$ & $56.6(4.9)$ \\
\hline \multicolumn{7}{|l|}{ Follow-up } \\
\hline Woman-years observed (I000s) & 3218.0 & 10270.0 & 3622.3 & 6608.7 & 2562.9 & | 3593.7 \\
\hline Number of incident cases: ICD-O-3 & 2469 & 6617 & 2532 & 4312 & 1797 & 9162 \\
\hline Number of incident cases: ICD-IO & 2152 & 5726 & 2191 & 3808 & 1487 & 7941 \\
\hline
\end{tabular}

Abbreviations: ICD-O-3 = International Classification of Diseases for Oncology 3rd edition; ICD-I0 = International Classification of Diseases IOth revision. ${ }^{\text {a }} \geqslant 0.5$ drinks per week, in units equivalent to $10 \mathrm{~g}$ pure alcohol. bHighest within-study tertile of the 199/ Townsend deprivation index for the census enumeration district or output area of the home address. 
Table 3 Association of alcohol drinking with risk of haematological malignancies

\begin{tabular}{|c|c|c|c|c|c|c|c|c|c|c|c|c|c|c|}
\hline \multirow[b]{2}{*}{ Alcohol $^{\mathrm{a}}$} & \multirow{2}{*}{$\begin{array}{c}\text { All } \\
\text { women } \\
\text { Cases }\end{array}$} & \multicolumn{3}{|c|}{ Non-drinkers } & \multicolumn{2}{|c|}{$\begin{array}{c}0.5-<3 \\
\text { drinks per } \\
\text { week }\end{array}$} & \multicolumn{3}{|c|}{$\begin{array}{c}3-<7 \\
\text { drinks per } \\
\text { week }\end{array}$} & \multicolumn{3}{|c|}{$\geqslant 7$ drinks per week } & \multicolumn{2}{|c|}{$\begin{array}{l}\text { Trend among } \\
\text { drinkers }\end{array}$} \\
\hline & & Cases & $\mathbf{R R}$ & $95 \% \mathrm{Cl}$ & Cases & Ref & Cases & $\mathbf{R R}$ & $95 \% \mathrm{Cl}$ & Cases & $\mathbf{R R}$ & $95 \% \mathrm{Cl}$ & Cases & $P_{\text {trend }}$ \\
\hline \multicolumn{15}{|l|}{ All haematological malignancies } \\
\hline ICD-O-3 classification & 9086 & 2469 & 1.05 & $0.99,1.10$ & 3364 & 1.00 & 1384 & 0.91 & $0.85,0.96$ & 1869 & 0.90 & $0.85,0.95$ & 6617 & $<0.001$ \\
\hline ICD-I0 classification & 7878 & 2152 & 1.06 & $1.00,1.12$ & 2924 & 1.00 & $120 \mid$ & 0.90 & $0.84,0.96$ & $|60|$ & 0.89 & $0.83,0.94$ & 5726 & $<0.001$ \\
\hline \multicolumn{15}{|l|}{$\begin{array}{l}\text { Subgroups of ICD-O-3 classification } \\
\text { ICD-O-3 haematological malignancies }\end{array}$} \\
\hline Lymphoid & 6990 & 1912 & 1.06 & $1.00,1.12$ & 2602 & 1.00 & 1067 & 0.90 & $0.84,0.97$ & 1409 & 0.88 & $0.82,0.94$ & 5078 & $<0.001$ \\
\hline Myeloid & 2053 & 546 & 1.01 & $0.91,1.14$ & 745 & 1.00 & 311 & 0.93 & $0.81,1.06$ & 451 & 0.99 & $0.88,1.11$ & 1507 & 0.8 \\
\hline \multicolumn{15}{|l|}{ ICD-O-3 lymphoid malignancies } \\
\hline Hodgkin lymphoma & 281 & 108 & 1.70 & $1.27,2.26$ & 85 & 1.00 & 31 & 0.79 & $0.53,1.20$ & 57 & 1.06 & $0.76,1.49$ & 173 & 0.6 \\
\hline Mature B cell & 5056 & 1344 & 1.01 & $0.94,1.08$ & 1925 & 1.00 & 763 & 0.87 & $0.80,0.95$ & 1024 & 0.87 & $0.81,0.94$ & 3712 & $<0.001$ \\
\hline Mature $\mathrm{T}$ cell & 196 & 40 & 0.78 & $0.53,1.15$ & 71 & 1.00 & 35 & 1.04 & $0.70,1.57$ & 50 & 1.05 & $0.73,1.52$ & 156 & 0.7 \\
\hline Other/unspecified lymphoid & 1457 & 420 & 1.18 & $1.03,1.34$ & 521 & 1.00 & 238 & 1.00 & $0.85,1.16$ & 278 & 0.84 & $0.73,0.98$ & 1037 & 0.02 \\
\hline \multicolumn{15}{|l|}{ ICD-O-3 mature B-cell malignancies } \\
\hline Diffuse large B-cell lymphoma & 1145 & 331 & 1.07 & $0.93,1.24$ & 443 & 1.00 & 153 & 0.77 & $0.64,0.92$ & 218 & 0.82 & $0.69,0.96$ & 814 & 0.01 \\
\hline Follicular lymphoma & $102 \mid$ & 255 & 0.96 & $0.82,1.13$ & 389 & 1.00 & 171 & 0.94 & $0.78,1.13$ & 206 & 0.82 & $0.69,0.98$ & 766 & 0.02 \\
\hline Plasma cell neoplasms & 1506 & 406 & 1.00 & $0.88,1.14$ & 587 & 1.00 & 223 & 0.84 & $0.72,0.98$ & 290 & 0.82 & $0.71,0.95$ & 1100 & 0.006 \\
\hline CLL/SLL & 911 & 245 & 1.10 & $0.93,1.30$ & 327 & 1.00 & 148 & 1.00 & $0.82,1.22$ & 191 & 0.97 & $0.81,1.16$ & 666 & 0.6 \\
\hline Other/unspecified mature B-cell & 473 & 107 & 0.83 & $0.65,1.06$ & 179 & 1.00 & 68 & 0.84 & $0.64,1.12$ & 119 & $|| \mid$. & $0.88,1.40$ & 366 & 0.4 \\
\hline \multicolumn{15}{|l|}{ ICD-O-3 myeloid malignancies ${ }^{c}$} \\
\hline Acute myeloid leukaemia & 613 & 165 & 0.99 & $0.81,1.21$ & 233 & 1.00 & 89 & 0.84 & $0.66,1.08$ & 126 & 0.89 & $0.71,1.11$ & 448 & 0.3 \\
\hline Myeloproliferative/myelodysplastic & 1415 & 372 & 1.02 & $0.89,1.17$ & 503 & 1.00 & 220 & 0.98 & $0.83,1.15$ & 320 & 1.03 & $0.89,1.19$ & 1043 & 0.7 \\
\hline
\end{tabular}

Subgroups of ICD-10 classification

ICD- IO haematological malignancies

Hodgkin lymphoma

Non-Hodgkin lymphoma

Myeloma

Leukaemia

$\begin{array}{rrrrrrrrr}281 & 108 & 1.70 & 1.27,2.26 & 85 & 1.00 & 31 & 0.79 & 0.53,1.20 \\ 4216 & 1132 & 1.02 & 0.95,1.11 & 1593 & 1.00 & 652 & 0.89 & 0.82,0.98 \\ 1584 & 422 & 0.99 & 0.88,1.13 & 616 & 1.00 & 231 & 0.83 & 0.72,0.97 \\ 1797 & 490 & 1.12 & 0.99,1.26 & 630 & 1.00 & 287 & 1.01 & 0.87,1.16\end{array}$

$\begin{array}{rrrr}57 & 1.06 & 0.76,1.49 & 173 \\ 839 & 0.84 & 0.77,0.92 & 3084 \\ 315 & 0.85 & 0.74,0.98 & 1162 \\ 390 & 1.01 & 0.89,1.15 & 1307\end{array}$

0.6

$<0.001$

0.02

0.9

Abbreviations: Cases = number of incident cases; $\mathrm{Cl}=$ confidence interval; CLL/SLL = chronic lymphocytic leukaemia/small lymphocytic lymphoma; ICD-O-3 = International Classification of Diseases for Oncology 3rd edition; ICD-IO = International Classification of Diseases 1 Oth revision; $P_{\text {trend }}=$ result of test for categorical trend per $10 \mathrm{~g}$ per day; Ref = referent; $R R=$ relative risk. Myeloproliferative/myelodysplastic disease includes chronic myeloid leukaemia. ${ }^{a}$ Reported alcohol consumption at recruitment, in units of approximately $10 \mathrm{~g}$ pure alcohol. RR estimates are adjusted for body mass index, height, smoking and socioeconomic status, and stratified by cancer registry region. Follow-up starts at recruitment. 'bxcludes 43 unspecified cases. ' Excludes 25 other/unspecified cases.

(1.27-2.26); Table 3); a similar result was obtained when the first 3 years of follow-up were excluded (data not shown).

Using the ICD-10 classification, there were significant decreasing trends for NHL and myeloma separately, and little evidence of association for leukaemia or Hodgkin lymphoma, although the test for heterogeneity of trends between diagnostic groups was not statistically significant $\left(P_{\text {het }}=0.06\right)$ (Figure 1$)$.

Excluding the first 3 years of follow-up made little difference to the trend estimates, but changed results from non-significant to significant for the tests of heterogeneity between lymphoid and myeloid malignancies $\left(P_{\text {het }}=0.01\right)$ and between mature B-cell subtypes $\left(P_{\text {het }}=0.01\right)$ (Table 5$)$.

\section{Smoking}

Using the ICD-O-3 classification, and taking women who had never smoked as the reference group, the estimated relative risk of haematological malignancy for frequent smokers $(\geqslant 15$ cigarettes per day) was 1.30 (1.20-1.40) (Table 4). There were statistically significant trends in both lymphoid (1.07 (1.03-1.12)) and myeloid (1.33 (1.24-1.42)) disease, with strong evidence of heterogeneity between these groups $\left(P_{\text {het }}<0.001\right)$ (Figure 1). There was also strong evidence of heterogeneity within lymphoid disease $\left(P_{\text {het }}<0.001\right)$, with statistically significant increasing trends for Hodgkin lymphoma (1.45 (1.22-1.72)) and mature T-cell malignancies
(1.38 (1.10-1.73)) but not for mature B-cell malignancies. There was heterogeneity between subgroups of myeloid malignancy $\left(P_{\text {het }}=0.001\right)$, with a statistically significant trend for myeloproliferative/myelodysplastic disease (1.42 (1.31-1.55)) but not for acute myeloid leukaemia (1.10 (0.96-1.26)). Comparing frequent smokers with never-smokers, the estimated relative risks of Hodgkin lymphoma, mature T-cell malignancies and myeloproliferative/myelodysplastic disease were each approximately doubled (2.19 (1.56-3.09), $2.09(1.33-3.26)$ and 1.98 (1.67-2.35), respectively) (Table 4).

Using the ICD-10 classification, there was strong evidence of heterogeneity between subgroups $\left(P_{\text {het }}<0.001\right)$, with statistically significant increasing trends for Hodgkin lymphoma and NHL but not for myeloma or leukaemia (Figure 1).

Excluding the first 3 years of follow-up made little difference to the trend estimates, and did not affect the conclusions of the tests for heterogeneity (Table 5).

\section{DISCUSSION}

\section{Alcohol}

In this cohort, most women who drank alcohol were moderate drinkers. Among the drinkers, greater alcohol intake was associated with significantly reduced risks of diffuse large B-cell lymphoma, follicular lymphoma and plasma cell neoplasms, 


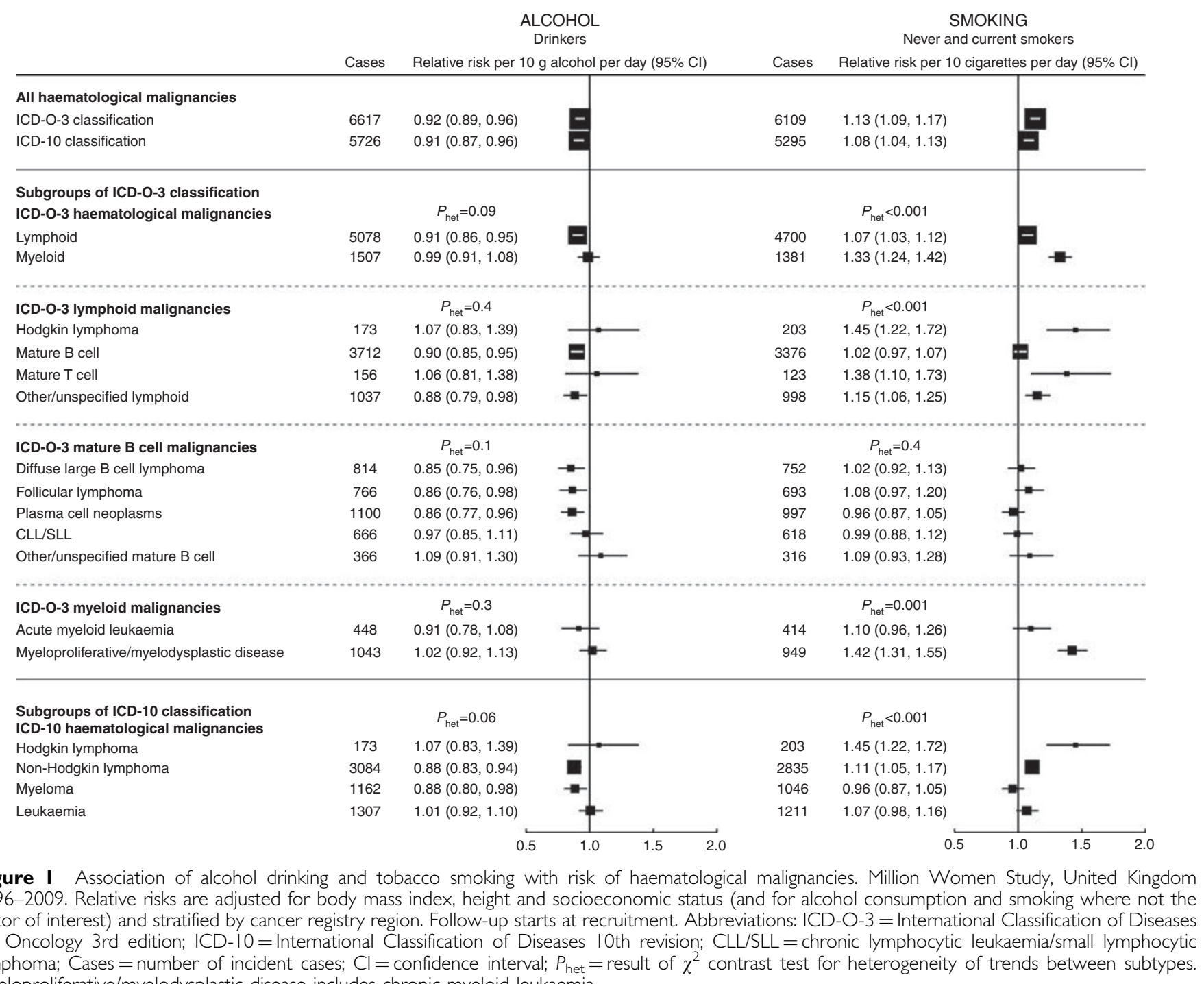

lymphoma; Cases = number of incident cases; $\mathrm{Cl}=$ confidence interval; $P_{\text {het }}=$ result of $\chi^{2}$ contrast test for heterogeneity of trends between subtypes. Myeloproliferative/myelodysplastic disease includes chronic myeloid leukaemia.

lymphoid and mature B-cell disease overall, but not other specified lymphoid subtypes or myeloid malignancies. Although there was no statistically significant heterogeneity between subtypes in the main analysis, significant heterogeneity between lymphoid and myeloid malignancies, and between mature B-cell subtypes, emerged when the first 3 years of follow-up were excluded. Thus some of the apparent lack of heterogeneity might be due to reverse causation. Using the ICD-10 classification, there were significant inverse associations with risks of NHL and myeloma, but not leukaemia or Hodgkin lymphoma.

Previous cohort studies have reported a statistically significant decreasing trend with greater alcohol intake among drinkers for diffuse large B-cell lymphoma (Lim et al, 2007; Troy et al, 2010), and no significant trend for Hodgkin lymphoma, follicular lymphoma, plasma cell neoplasms or any other non-Hodgkin subtype examined (Lim et al, 2007; Kanda et al, 2010; Troy et al, 2010), although one study found a near-significant decreasing trend for plasma cell neoplasms (Troy et al, 2010); comparable results from case-control studies were generally consistent with these findings (Morton et al, 2005b; Besson et al, 2006a, 2006b). In studies that estimated risks in drinkers relative to non-drinkers, similar results were obtained for lymphoid subtypes (Kanda et al, 2009; Chang et al, 2010) except for one observation of increased CLL/SLL risk in drinkers (Chang et al,
2010), and there was no trend in risk of acute myeloid leukaemia ( $\mathrm{Ma}$ et al, 2010) or myelodysplastic syndromes (Ma et al, 2009). A multicentre case-control study reported lower risk of Hodgkin lymphoma in ever-regular drinkers compared with never-regular drinkers, based on 222 cases (Besson et al, 2006a). In our study, with 281 cases in total, the risk of Hodgkin lymphoma was estimated to be lower among current occasional drinkers (at recruitment) than in current non-drinkers, but there was no significant trend in risk with increasing intake; excluding the first 3 years of follow-up did not change either of these findings.

Using a similar ICD-10 classification, an earlier analysis of data from the Million Women Study with 7.2 years of follow-up on average (Allen et al, 2009) reported a statistically significant inverse association in drinkers for NHL, but not for myeloma or leukaemia; the significant association with myeloma seen in the analysis reported here probably reflects the larger number of cases accumulated over a longer follow-up period. These results are broadly consistent with comparable findings from other cohort (Chiu et al, 1999; Klatsky et al, 2009) and case-control (Gorini et al, 2007a,b) studies.

Our findings strengthen existing evidence for an association of greater alcohol intake with reduced risk of diffuse large B-cell lymphoma among drinkers, and demonstrate similar associations 
Table 4 Association of tobacco smoking with risk of haematological malignancies

\begin{tabular}{|c|c|c|c|c|c|c|c|c|c|c|c|c|c|c|}
\hline \multirow[b]{2}{*}{ Smoking ${ }^{a}$} & \multirow{2}{*}{$\begin{array}{c}\text { All } \\
\text { women } \\
\text { Cases }\end{array}$} & \multicolumn{3}{|c|}{ Ex-smokers } & \multicolumn{2}{|c|}{$\begin{array}{l}\text { Never- } \\
\text { smokers }\end{array}$} & \multicolumn{3}{|c|}{$\begin{array}{c}<\text { I5 cigarettes per } \\
\text { day }\end{array}$} & \multicolumn{3}{|c|}{$\underset{\text { day }}{\geq 15 \text { cigarettes per }}$} & \multicolumn{2}{|c|}{$\begin{array}{c}\text { Trend among } \\
\text { never- or } \\
\text { current } \\
\text { smokers }\end{array}$} \\
\hline & & Cases & $\mathbf{R R}$ & $95 \% \mathrm{Cl}$ & Cases & Ref & Cases & $\mathbf{R R}$ & $95 \% \mathrm{Cl}$ & Cases & $\mathbf{R R}$ & $95 \% \mathrm{Cl}$ & Cases & $P_{\text {trend }}$ \\
\hline \multicolumn{15}{|l|}{ All haematological malignancies } \\
\hline ICD-O-3 classification & 8641 & 2532 & 1.09 & $1.04,1.15$ & 4312 & 1.00 & 896 & 1.13 & $1.05,1.22$ & 901 & 1.30 & $1.20,1.40$ & 6109 & $<0.001$ \\
\hline ICD- I0 classification & 7486 & 2191 & 1.07 & $1.01,1.13$ & 3808 & 1.00 & 747 & 1.06 & $0.98,1.15$ & 740 & 1.19 & $1.10,1.29$ & 5295 & $<0.001$ \\
\hline \multicolumn{15}{|l|}{$\begin{array}{l}\text { Subgroups of ICD-O-3 classification } \\
\text { ICD-O-3 haematological malignancies }\end{array}$} \\
\hline Lymphoid & 6638 & 1938 & 1.06 & $1.01,1.13$ & 3394 & 1.00 & 653 & 1.04 & $0.96,1.14$ & 653 & 1.18 & $1.09,1.29$ & 4700 & $<0.001$ \\
\hline Myeloid & 1963 & 582 & 1.19 & $1.07,1.32$ & 902 & 1.00 & 238 & 1.46 & $1.26,1.69$ & 241 & 1.69 & $1.46,1.96$ & $|38|$ & $<0.001$ \\
\hline \multicolumn{15}{|l|}{ ICD-O-3 lymphoid malignancies } \\
\hline Hodgkin lymphoma & 263 & 60 & 0.90 & $0.66,1.23$ & 122 & 1.00 & 31 & 1.30 & $0.87,1.94$ & 50 & 2.19 & $1.56,3.09$ & 203 & $<0.001$ \\
\hline Mature B cell & 4803 & 1427 & 1.07 & $1.00,1.14$ & 2498 & 1.00 & 452 & 0.98 & $0.89,1.09$ & 426 & 1.06 & $0.96,1.18$ & 3376 & 0.5 \\
\hline Mature $T$ cell & 184 & 61 & 1.38 & $0.98,1.93$ & 78 & 1.00 & 17 & 1.15 & $0.68,1.95$ & 28 & 2.09 & $1.33,3.26$ & 123 & 0.006 \\
\hline Other/unspecified lymphoid & 1388 & 390 & 1.05 & $0.93,1.19$ & 696 & 1.00 & 153 & 1.20 & $1.01,1.44$ & 149 & 1.31 & $1.10,1.58$ & 998 & 0.001 \\
\hline \multicolumn{15}{|l|}{ ICD-O-3 mature B-cell malignancies } \\
\hline Diffuse large B-cell lymphoma & 1084 & 332 & I.I I & $0.96,1.27$ & 560 & 1.00 & 99 & 0.97 & $0.78,1.21$ & 93 & 1.04 & $0.83,1.30$ & 752 & 0.7 \\
\hline Follicular lymphoma & 976 & 283 & 1.07 & $0.92,1.24$ & 497 & 1.00 & 94 & 1.00 & $0.80,1.25$ & 102 & 1.22 & $0.98,1.52$ & 693 & 0.1 \\
\hline Plasma cell neoplasms & 1425 & 428 & 1.08 & $0.96,1.22$ & 750 & 1.00 & 140 & 1.03 & $0.86,1.24$ & 107 & 0.91 & $0.74,1.12$ & 997 & 0.4 \\
\hline CLL/SLL & 870 & 252 & 1.02 & $0.87,1.19$ & 461 & 1.00 & 84 & 1.00 & $0.79,1.26$ & 73 & $1.0 \mid$ & $0.78,1.29$ & 618 & 0.9 \\
\hline Other/unspecified mature B cell & 448 & 132 & 1.02 & $0.82,1.27$ & 230 & 1.00 & 35 & 0.79 & $0.55,1.13$ & 51 & 1.32 & $0.96,1.80$ & 316 & 0.3 \\
\hline ICD-O-3 myeloid malignancies ${ }^{c}$ & & & & & & & & & & & & & & \\
\hline Acute myeloid leukaemia & 586 & 172 & 1.08 & $0.89,1.31$ & 291 & 1.00 & 70 & 1.29 & $0.99,1.68$ & 53 & 1.08 & $0.80,1.46$ & 414 & 0.2 \\
\hline $\begin{array}{l}\text { Myeloproliferative/myelodysplastic } \\
\text { disease }\end{array}$ & 1353 & 404 & 1.24 & $1.09,1.41$ & 602 & 1.00 & 163 & 1.52 & ।.27, I.8। & 184 & 1.98 & $1.67,2.35$ & 949 & $<0.00$ \\
\hline \multicolumn{15}{|l|}{$\begin{array}{l}\text { Subgroups of ICD- } 10 \text { classification } \\
\text { ICD- } 10 \text { haematological malignancies }\end{array}$} \\
\hline Hodgkin lymphoma & 263 & 60 & 0.90 & $0.66,1.23$ & 122 & 1.00 & 31 & 1.30 & $0.87,1.94$ & 50 & 2.19 & $1.56,3.09$ & 203 & $<0.001$ \\
\hline Non-Hodgkin lymphoma & 4011 & 1176 & 1.08 & $1.01,1.16$ & 2024 & 1.00 & 392 & 1.05 & $0.94,1.17$ & 419 & 1.26 & $1.14,1.4 \mid$ & 2835 & $<0.001$ \\
\hline Myeloma & 1496 & 450 & 1.08 & $0.96,1.21$ & 789 & 1.00 & 144 & 1.01 & $0.84,1.21$ & 113 & 0.92 & $0.75,1.12$ & 1046 & 0.3 \\
\hline Leukaemia & 1716 & 505 & 1.06 & $0.95,1.19$ & 873 & 1.00 & 180 & 1.12 & $0.95,1.32$ & 158 & 1.11 & $0.93,1.32$ & 1211 & 0.1 \\
\hline
\end{tabular}

for two further subtypes of lymphoid disease: follicular lymphoma and plasma cell neoplasms. Further work is needed to elucidate potential biological mechanisms; for example, the role of chronic inflammation (Smedby et al, 2008; Chang et al, 2010).

\section{Smoking}

We found statistically significant increasing trends in risk of Hodgkin lymphoma, mature T-cell malignancies and myeloproliferative/ myelodysplastic disease with increasing current cigarette consumption relative to never-smokers (approximately double risk for women who reported smoking $\geqslant 15$ cigarettes per day), but no significant trends for mature B-cell malignancy or any of its subtypes. Tests for heterogeneity between diagnostic subgroups were highly significant. The trend estimate for acute myeloid leukaemia, although above unity, was not statistically significant. Using the ICD-10 classification, there were significant increasing trends for Hodgkin lymphoma and NHL but not for myeloma or leukaemia. Excluding the first 3 years of follow-up did not affect these conclusions.

Recent cohort studies, also comparing current smokers with never-smokers, have reported statistically significant associations for Hodgkin lymphoma (Lim et al, 2007; Nieters et al, 2008), myelodysplastic syndromes (Ma et al, 2009) and acute myeloid leukaemia (Fernberg et al, 2007; Ma et al, 2010), but no association for T-cell malignancies or any other non-Hodgkin subtype examined (Lim et al, 2007; Nieters et al, 2008; Troy et al, 2010; Lu et al, 2011). Results from case-control studies were similar: comparing current with never-smokers there were significant positive associations for Hodgkin lymphoma (Besson et al, 2006a) and acute myeloid leukaemia (Kasim et al, 2005), but no association for T-cell malignancies or any other non-Hodgkin subtype examined (Morton et al, 2005a; Besson et al, 2006b) except for one positive association for follicular lymphoma (Morton et al, 2005a); comparing ever- with never-smokers there were significant positive associations for myelodysplastic syndromes (Nisse et al, 2001; Strom et al, 2005). Trend analyses including former smokers suggested (in a cohort study) an inverse association with follicular lymphoma (Lim et al, 2007) and (in a case-control study) a positive association with Hodgkin lymphoma (Kanda et al, 2009).

Our findings support existing evidence for associations of smoking with Hodgkin lymphoma and myelodysplastic syndromes (a subset of myeloproliferative/myelodysplastic disease), and demonstrate a similar association for mature T-cell malignancies. Tobacco smoke contains benzene and other known leukaemogens, and it has been concluded that there is 'sufficient evidence in humans' that 
Table 5 Association of alcohol drinking and tobacco smoking with risk of haematological malignancies: trend analysis excluding the first 3 years of follow-up

\begin{tabular}{|c|c|c|c|c|c|c|c|c|}
\hline \multirow[b]{2}{*}{ Excluding first 3 years of follow-up } & \multicolumn{4}{|c|}{$\begin{array}{l}\text { Alcohol } \\
\text { Trend among drinkers }\end{array}$} & \multicolumn{4}{|c|}{$\begin{array}{c}\text { Smoking } \\
\text { Trend among never- or current smokers }{ }^{\mathrm{b}}\end{array}$} \\
\hline & Cases & $\mathbf{R R}$ & $95 \% \mathrm{Cl}$ & $P_{\text {trend }}$ & Cases & $\mathbf{R R}$ & $95 \% \mathrm{Cl}$ & $P_{\text {trend }}$ \\
\hline \multicolumn{9}{|l|}{ All haematological malignancies } \\
\hline ICD-O-3 classification & 5235 & 0.94 & $0.89,0.98$ & 0.006 & 4825 & 1.14 & $1.09,1.18$ & $<0.001$ \\
\hline ICD-I0 classification & 4528 & 0.92 & $0.87,0.97$ & 0.001 & 4170 & 1.07 & $1.03,1.12$ & 0.001 \\
\hline \multicolumn{9}{|l|}{ Subgroups of ICD-O-3 classification } \\
\hline Lymphoid & 4031 & 0.91 & $0.86,0.96$ & $<0.001$ & 3721 & 1.07 & $1.02,1.12$ & 0.006 \\
\hline Myeloid & 1177 & 1.04 & $0.95,1.15$ & 0.4 & 1082 & 1.36 & $1.26,1.47$ & $<0.001$ \\
\hline ICD-O-3 lymphoid malignancies & \multicolumn{4}{|c|}{$P_{\text {het }}=0.2$} & \multicolumn{4}{|c|}{$P_{\text {het }}<0.001$} \\
\hline Hodgkin lymphoma & 129 & 1.07 & $0.79,1.44$ & 0.7 & 152 & 1.57 & $1.29,1.90$ & $<0.001$ \\
\hline Mature B cell & 3045 & 0.90 & $0.84,0.95$ & $<0.001$ & 2774 & 1.00 & $0.95,1.06$ & 0.9 \\
\hline Mature T cell & 118 & 1.19 & $0.88,1.61$ & 0.3 & 88 & 1.34 & $1.02,1.77$ & 0.03 \\
\hline Other/unspecified lymphoid & 739 & \multicolumn{2}{|c|}{$P_{\text {het }}=0.01$} & 0.05 & 707 & 1.17 & $1.06,1.30$ & 0.002 \\
\hline CLL/SLL & 540 & 0.97 & $0.83,1.12$ & 0.6 & 494 & 0.96 & $0.84,1.10$ & 0.6 \\
\hline Other/unspecified mature B cell & 315 & 1.19 & $0.99,1.43$ & 0.07 & 268 & 1.10 & $0.93,1.30$ & 0.3 \\
\hline ICD-O-3 myeloid malignancies ${ }^{c}$ & \multicolumn{4}{|c|}{$P_{\text {het }}=0.4$} & \multicolumn{4}{|c|}{$P_{\text {het }}<0.001$} \\
\hline Acute myeloid leukaemia & 343 & 0.98 & $0.81,1.17$ & 0.8 & 311 & 1.07 & $0.91,1.26$ & 0.4 \\
\hline Myeloproliferative/myelodysplastic disease & 821 & 1.07 & $0.95,1.21$ & 0.2 & 759 & 1.48 & $1.35,1.62$ & $<0.001$ \\
\hline \multicolumn{9}{|l|}{ Subgroups of ICD-1 0 classification } \\
\hline ICD-I 0 haematological malignancies & \multicolumn{4}{|c|}{$P_{\text {het }}=0.06$} & \multicolumn{4}{|c|}{$P_{\text {het }}<0.001$} \\
\hline Hodgkin lymphoma & 129 & 1.07 & $0.79,1.44$ & 0.7 & 152 & 1.57 & $1.29,1.90$ & $<0.001$ \\
\hline Non-Hodgkin lymphoma & 2435 & 0.89 & $0.83,0.96$ & 0.001 & 2227 & 1.12 & $1.05,1.18$ & $<0.001$ \\
\hline Myeloma & 947 & 0.86 & $0.76,0.96$ & 0.008 & 856 & 0.92 & $0.83,1.02$ & 0.1 \\
\hline Leukaemia & 1017 & 1.03 & $0.93,1.14$ & 0.6 & 935 & 1.03 & $0.94,1.13$ & 0.5 \\
\hline
\end{tabular}

Abbreviations: Cases, number of incident cases; Cl, confidence interval; CLL/SLL, chronic lymphocytic leukaemia/small lymphocytic lymphoma; ICD-O-3, International Classification of Diseases for Oncology 3rd edition; ICD- I0, International Classification of Diseases IOth revision; $P_{\text {het }}$ result of $\chi^{2}$ contrast test for heterogeneity of trends between subtypes; Ref, referent; $P_{\text {trend, }}$ result of test for categorical trend; RR, relative risk. Myeloproliferative/myelodysplastic disease includes chronic myeloid leukaemia.

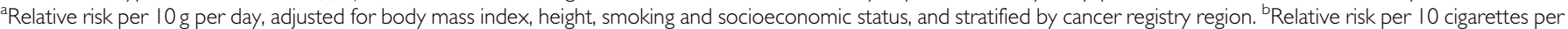
day, adjusted for body mass index, height, alcohol consumption and socioeconomic status, and stratified by cancer registry region. 'Excludes other/unspecified cases.

tobacco smoking causes myeloid (but not lymphoid) leukaemia (IARC, 2002). Hence, smoking is a plausible cause of myeloproliferative/myelodysplastic disease, which includes chronic myeloid leukaemia and various myeloid pre-leukaemic conditions. An association with $\mathrm{T}$-cell disease has not been reported before, to our knowledge, perhaps because this is a relatively rare diagnostic group. It has been suggested that smoking might impair the T-cellmediated immune response to Epstein-Barr virus infection, a putative causal factor for Hodgkin lymphoma (Nieters et al, 2008), and it is tempting to speculate that such a process might also promote T-cell malignancies.

\section{Strengths and limitations}

This very large prospective study clarifies and extends existing evidence for associations of subtypes of haematological malignancy with alcohol and tobacco consumption, using two different current classification systems. Exposures were reported by the study participants at recruitment, and follow-up for death, emigration and cancer registration was virtually complete. Estimates were mutually adjusted for alcohol and smoking, socioeconomic status, body mass index and height. Reverse causation is an unlikely explanation for the associations seen, as excluding the first 3 years of follow-up did not qualitatively change the results.

Although the analysis was stratified by cancer registry region of residence at recruitment, variation in diagnostic and coding practice remains a possible source of bias. The registries adopted ICD-O-3 during the study period, at times that differed between regions, and previous ascertainment of myeloproliferative/myelodysplastic disease is likely to have been incomplete (Office for National Statistics, 2010). Subclassification of lymphoma may sometimes have been inaccurate (Clarke et al, 2004, 2006) and was often imprecise. Conceivably, time to diagnosis might be associated with the factors of interest: for example, causes of mediastinal symptoms of Hodgkin lymphoma might perhaps be investigated more rapidly in smokers because of the well-known risk of lung disease in smokers. However, the strong and highly significant associations reported are unlikely to be due to coding problems, or to chance.

\section{CONCLUSIONS}

Relative risks associated with alcohol and tobacco consumption among middle-aged women in the United Kingdom were estimated for subtypes of haematological malignancy. Among predominantly moderate drinkers, greater alcohol intake was associated with reduced risk of lymphoid malignancies: in particular, diffuse large B-cell lymphoma, consistent with previous reports, and follicular lymphoma and plasma cell neoplasms (not previously reported, to our knowledge). Cigarette smoking was associated with increased risk of Hodgkin lymphoma, consistent with previous reports, 
mature T-cell malignancies (not previously reported, to our knowledge) and myeloproliferative/myelodysplastic disease (previously reported for myelodysplastic syndromes, but not for the grouping used here).

\section{REFERENCES}

Allen NE, Beral V, Casabonne D, Kan SW, Reeves GK, Brown A, Green J (2009) Moderate alcohol intake and cancer incidence in women. J Natl Cancer Inst 101(5): 296-305

Besson H, Brennan P, Becker N, De Sanjose S, Nieters A, Font R, Maynadie M, Foretova L, Cocco PL, Staines A, Vornanen M, Boffetta P (2006a) Tobacco smoking, alcohol drinking and Hodgkin's lymphoma: a European multi-centre case-control study (EPILYMPH). Br J Cancer 95(3): 378-384

Besson H, Brennan P, Becker N, Nieters A, De Sanjose S, Font R, Maynadie M, Foretova L, Cocco PL, Staines A, Vornanen M, Boffetta P (2006b) Tobacco smoking, alcohol drinking and non-Hodgkin's lymphoma: A European multicenter case-control study (Epilymph). Int J Cancer 119(4): 901-908

Chang ET, Clarke CA, Canchola AJ, Lu Y, Wang SS, Ursin G, West DW, Bernstein L, Horn-Ross PL (2010) Alcohol consumption over time and risk of lymphoid malignancies in the California Teachers Study cohort. Am J Epidemiol 172(12): 1373-1383

Chiu BCH, Cerhan JR, Gapstur SM, Sellers TA, Zheng W, Lutz CT, Wallace RB, Potter JD (1999) Alcohol consumption and non-Hodgkin lymphoma in a cohort of older women. Br J Cancer 80(9): 1476-1482

Clarke CA, Glaser SL, Dorfman RF, Bracci PM, Eberle E, Holly EA (2004) Expert review of non-Hodgkin's lymphomas in a population-based cancer registry: reliability of diagnosis and subtype classifications. Cancer Epidemiol Biomarkers Prev 13(1): 138-143

Clarke CA, Undurraga DM, Harasty PJ, Glaser SL, Morton LM, Holly EA (2006) Changes in cancer registry coding for lymphoma subtypes: reliability over time and relevance for surveillance and study. Cancer Epidemiol Biomarkers Prev 15(4): 630-638

Fernberg P, Odenbro A, Bellocco R, Boffetta P, Pawitan Y, Zendehdel K, Adami J (2007) Tobacco use, body mass index, and the risk of leukemia and multiple myeloma: a nationwide cohort study in Sweden. Cancer Res 67(12): 5983-5986

Gorini G, Stagnaro E, Fontana V, Miligi L, Ramazzotti V, Amadori D, Rodella S, Tumino R, Crosignani P, Vindigni C, Fontana A, Vineis P, Seniori Costantini A (2007a) Alcohol consumption and risk of Hodgkin's lymphoma and multiple myeloma: a multicentre case-control study. Ann Oncol 18(1): 143-148

Gorini G, Stagnaro E, Fontana V, Miligi L, Ramazzotti V, Nanni O, Rodella S, Tumino R, Crosignani P, Vindigni C, Fontana A, Vineis P, Costantini AS (2007b) Alcohol consumption and risk of leukemia: A multicenter case-control study. Leuk Res 31(3): 379-386

Green J, Cairns BJ, Casabonne D, Wright FL, Reeves G, Beral V (2011) Height and cancer incidence in the Million Women Study: prospective cohort, and meta-analysis of prospective studies of height and total cancer risk. Lancet Oncol 12(8): 785-794

IARC (2002) IARC Monographs on the Evaluation of Carcinogenic Risks to Humans. Tobacco Smoke and Involuntary Smoking. Vol. 83. International Agency for Research on Cancer: Lyon

Jaffe ES, Harris NL, Stein H, Vardiman JW (eds) (2001) World Health Organization Classification of Tumours: Pathology and Genetics of Tumours of Haematopoietic and Lymphoid Tissues. IARC Press: Lyon, France

Kanda J, Matsuo K, Inoue M, Iwasaki M, Sawada N, Shimazu T, Yamaji T, Sasazuki S, Tsugane S, Japan Public Health Center-based Prospective Study G (2010) Association of alcohol intake with the risk of malignant lymphoma and plasma cell myeloma in Japanese: a population-based cohort study (Japan Public Health Centerbased Prospective Study). Cancer Epidemiol Biomarkers Prev 19(2): 429-434

Kanda J, Matsuo K, Kawase T, Suzuki T, Ichinohe T, Seto M, Morishima Y, Tajima K, Tanaka H (2009) Association of alcohol intake and smoking with malignant lymphoma risk in Japanese: a hospital-based case-control study at Aichi Cancer Center. Cancer Epidemiol Biomarkers Prev 18(9): 2436-2441

Kasim K, Levallois P, Abdous B, Auger P, Johnson KC (2005) Lifestyle factors and the risk of adult leukemia in Canada. Cancer Causes Control 16(5): 489-500
Klatsky AL, Li Y, Baer D, Armstrong MA, Udaltsova N, Friedman GD (2009) Alcohol Consumption and Risk of Hematologic Malignancies. Ann Epidemiol 19(10): 746-753

Lim U, Morton LM, Subar AF, Baris D, Stolzenberg-Solomon R, Leitzmann M, Kipnis V, Mouw T, Carroll L, Schatzkin A, Hartge P (2007) Alcohol, smoking, and body size in relation to incident Hodgkin's and nonHodgkin's lymphoma risk. Am J Epidemiol 166(6): 697-708

Lu Y, Wang SS, Reynolds P, Chang ET, Ma H, Sullivan-Halley J, Clarke CA, Bernstein L (2011) Cigarette smoking, passive smoking, and nonHodgkin lymphoma risk: evidence from the California Teachers Study. Am J Epidemiol 174(5): 563-573

Ma X, Lim U, Park Y, Mayne ST, Wang R, Hartge P, Hollenbeck AR, Schatzkin A (2009) Obesity, lifestyle factors, and risk of myelodysplastic syndromes in a large US cohort. Am J Epidemiol 169(12): 1492-1499

Ma X, Park Y, Mayne ST, Wang R, Sinha R, Hollenbeck AR, Schatzkin A, Cross AJ (2010) Diet, lifestyle, and acute myeloid leukemia in the NIHAARP cohort. Am J Epidemiol 171(3): 312-322

Macmahon S, Peto R, Cutler J, Collins R, Sorlie P, Neaton J, Abbott R, Godwin J, Dyer A, Stamler J (1990) Blood-pressure, stroke, and coronary heart-disease.1. Prolonged differences in blood-pressure - prospective observational studies corrected for the regression dilution bias. Lancet 335(8692): 765-774

Morton LM, Hartge P, Holford TR, Holly EA, Chiu BCH, Vineis P, Stagnaro E, Willett EV, Franceschi S, La Vecchia C, Hughes AM, Cozen W, Davis S, Severson RK, Bernstein L, Mayne ST, Dee FR, Cerhan JR, Zheng TZ (2005a) Cigarette smoking and risk of non-Hodgkin lymphoma: A pooled analysis from the international lymphoma epidemiology consortium (InterLymph). Cancer Epidemiol Biomarkers Prev 14(4): 925-933

Morton LM, Turner JJ, Cerhan JR, Linet MS, Treseler PA, Clarke CA, Jack A, Cozen W, Maynadié M, Spinelli JJ, Costantini AS, Rüdiger T, Scarpa A, Zheng T, Weisenburger DD (2007) Proposed classification of lymphoid neoplasms for epidemiologic research from the Pathology Working Group of the International Lymphoma Epidemiology Consortium (InterLymph). Blood 110(2): 695-708

Morton LM, Zheng T, Holford TR, Holly EA, Chiu BC, Costantini AS, Stagnaro E, Willett EV, Dal Maso L, Serraino D, Chang ET, Cozen W, Davis S, Severson RK, Bernstein L, Mayne ST, Dee FR, Cerhan JR, Hartge $\mathrm{P}$, InterLymph C (2005b) Alcohol consumption and risk of non-Hodgkin lymphoma: a pooled analysis. Lancet Oncology 6(7): 469-476

National Cancer Institute. Surveillance Reasearch Program: ICD conversion programs. seer.cancer.gov/tools/conversion/ICDO2-3.xls (accessed 25 March 2011)

Nieters A, Rohrmann S, Becker N, Linseisen J, Ruediger T, Overvad K, Tjonneland A, Olsen A, Allen NE, Travis RC, Bingham S, Khaw KT, Ardanaz E, Redondo ML, Basterrechea M, Martinez C, Tormo MJ, Rosso S, Tagliabue G, Masala G, Mattiello A, Tumino R, Boeing H, Bergmann M, Kaaks R, Trichopoulou A, Trichopoulos D, Peeters PH, Bueno-de-Mesquita B, Boffetta P, Brennan P, Ferrari P, Neasham D, Lund E, Berglund G, Manjer J, Hallmans G, Johansson I, Vineis P, Riboli E (2008) Smoking and lymphoma risk in the European prospective investigation into cancer and nutrition. Am J Epidemiol 167(9): 1081-1089

Nisse C, Haguenoer JM, Grandbastien B, Preudhomme C, Fontaine B, Brillet JM, Lejeune R, Fenaux P (2001) Occupational and environmental risk factors of the myelodysplastic syndromes in the North of France. $\mathrm{Br} J$ Haematol 112(4): 927-935

Office for National Statistics (2003) The National Diet and Nutrition Survey: Adults Aged 19 to 64 years. Vol. 2. HMSO: London

Office for National Statistics (2010) Cancer Statistics Registrations, England (Series MB1). No. 39, 2008. HMSO: London

Reeves GK, Pirie K, Beral V, Green J, Spencer E, Bull D (2007) Cancer incidence and mortality in relation to body mass index in the Million Women Study: Cohort study. Br Med J 335(7630): 1134-1139

Sant M, Allemani C, Tereanu C, De Angelis R, Capocaccia R, Visser O, Marcos-Gragera R, Maynadié M, Simonetti A, Lutz JM, Berrino F, Hackl M, Holub J, Maynadie M, Holleczek B, Tryggvadottir L, Comber H, Bellù F, Giacomin A, Ferretti S, Crocetti E, Serraino D, Vercelli M, Federico M, Fusco M, Michiara M, Tumino R, Mangone L, Falcini F, Iannelli A, 
Budroni M, Zanetti R, Piffer S, La Rosa F, Zambon P, Sowe S, England K, Langmark F, Rachtan J, Mezyk R, Zwierko M, Ondrusova M, PrimicŽakelj M, Khan S, Jundt G, Usel M, Ess SM, Bordoni A, Otter R, Coebergh JW, Siesling S, Greenberg D, Easey N, Roche M, Lawrence G, Gavin A, Brewster DH, Steward J (2010) Incidence of hematologic malignancies in Europe by morphologic subtype: Results of the HAEMACARE project. Blood 116(19): 3724-3734

Smedby KE, Askling J, Mariette X, Baecklund E (2008) Autoimmune and inflammatory disorders and risk of malignant lymphomas-an update. J Intern Med 264(6): 514-527

Smith-Warner SA, Spiegelman D, Ritz J, Albanes D, Beeson WL, Bernstein L, Berrino F, van den Brandt PA, Buring JE, Cho E, Colditz GA, Folsom AR, Freudenheim JL, Giovannucci E, Goldbohm RA, Graham S, Harnack L, Horn-Ross PL, Krogh V, Leitzmann MF, McCullough ML, Miller AB, Rodriguez C, Rohan TE, Schatzkin A, Shore R, Virtanen M, Willett WC, Wolk A, Zeleniuch-Jacquotte A, Zhang SM, Hunter DJ (2006) Methods for pooling results of epidemiologic studies: the Pooling Project of Prospective Studies of Diet and Cancer. Am J Epidemiol 163(11): 1053-1064

StataCorp (2009) Stata Statistical Software. Release 11
Strom SS, Gu Y, Gruschkus SK, Pierce SA, Estey EH (2005) Risk factors of myelodysplastic syndromes: A case-control study. Leukemia 19(11): 1912-1918

Swerdlow SCE, Harris N (2008) World Health Organization Classification of Tumours of Haematopoietic and Lymphoid Tissues. IARC Press: Lyon, France

Townsend PPP, Beattie A (1988) Health and deprivation: inequality and the North. Croom Helm: London

Troy JD, Hartge P, Weissfeld JL, Oken MM, Colditz GA, Mechanic LE, Morton LM (2010) Associations between anthropometry, cigarette smoking, alcohol consumption, and non-Hodgkin lymphoma in the Prostate, Lung, Colorectal, and Ovarian Cancer Screening Trial. Am J Epidemiol 171(12): 1270-1281

Turner JJ, Morton LM, Linet MS, Clarke CA, Kadin ME, Vajdic CM, Monnereau A, Maynadié M, Chiu BCH, Marcos-Gragera R, Costantini AS, Cerhan JR, Weisenburger DD (2010) InterLymph hierarchical classification of lymphoid neoplasms for epidemiologic research based on the WHO classification (2008): Update and future directions. Blood 116(20): e90-e98

This work is published under the standard license to publish agreement. After 12 months the work will become freely available and the license terms will switch to a Creative Commons Attribution-NonCommercial-Share Alike 3.0 Unported License. 